\title{
Book Proposal on Biomimetic Tissue Simulants
}

\author{
Arnab Chanda
}

email: arnab1232@gmail.com

\begin{abstract}
Soft tissue surrogate based test dummies are used across industries to simulate real life accidents. To date, there are a wide range of surrogates available in the market, including gels, elastomers, and animal tissues, which are backdated and have mechanical properties very different from actual human tissues. However, in academic research, biofidelic soft tissue surrogates have evolved in the last two decades, but have lacked technology transfer. This book aims to bridge the gap between the industry and academia with the state of the art in soft tissue surrogate research. Surrogates are presented with respect to skin, muscles, brain tissue, arteries, and female pelvis. Fabrication techniques, mechanical testing, and test results required for reproducing these surrogates are discussed. Also, characterization methodologies and limitations of each type of surrogate are presented, for their use in both experimental and computational research. Some major industries which can use these biofidelic surrogates are car manufacturers, prosthetics and orthotics designers, ballistic testing facilities, military and sports equipment manufacturers. Also, hospitals and medical centres can take advantage of these synthetic surrogates over actual tissues for surgical training with minimal biosafety approvals and ethical issues.
\end{abstract}

\section{Introduction}

These references will get you started. Then search the citations within the references and the ones citing the article to make sure. Soft tissue surrogates currently used in the industry for biomechanical testing are backdated and very different from live human tissues with respect to mechanical properties. This goal of this book is to present the state of the art in development and characterization of soft tissue surrogates in academic research for their immediate translation into the industry, for both experimental and computational testing. (Arnab Chanda, Unnikrishnan, Roy, et al., 2015)(Arnab Chanda, Callaway, Clifton, et al., 2018)(Arnab Chanda \& Unnikrishnan, 2019)(A. Chanda \& Ghoneim, 2015)(Arnab Chanda, Graeter, et al., 2015)(Arnab Chanda et al., 2016a)(Arnab Chanda \& Unnikrishnan, 2017)(Arnab Chanda, Unnikrishnan, Flynn, et al., 2017)(Gupta et al., 2020)(Arnab Chanda \& Unnikrishnan, 2016)(Arnab Chanda \& Callaway, 2018)(Arnab Chanda, Unnikrishnan, et al., 2018)(Arnab Chanda, Meyer, et al., 2017)(Arnab Chanda, Unnikrishnan, \& Flynn, 2015)(Arnab Chanda, Ruchti, \& Unnikrishnan, 2018)(Arnab Chanda, Flynn, et al., 2018)(Arnab Chanda \& Unnikrishnan, 2018b)(Arnab Chanda et al., 2016b)(Ghoneim \& Chanda, 2013)(Arnab Chanda, Unnikrishnan, \& Lackey, 2017)(Kushner, 1992)(Arnab Chanda \& Curry, 2018)(Arnab Chanda, 2017)(Arnab Chanda, 2018a)(Arnab Chanda, Jones, \& Beschorner, 2018)(Arnab Chanda \& Unnikrishnan, 2018c)(Arnab Chanda \& Graeter, 2018)(Arnab Chanda, 2018b)(Arnab Chanda \& Unnikrishnan, 2015e)(Sundaram et al., 2020)(Arnab Chanda et al., 2019)(Arnab Chanda \& Unnikrishnan, 2018a)(Arnab Chanda, 
Ruchti, \& Upchurch, 2018)(Arnab Chanda, n.d.)(Beschorner et al., 2020)(Arnab Chanda, Chatterjee, et al., 2020)(Arnab; Chanda et al., 2019)(Arnab Chanda, Unnikrishnan, et al., 2020)(Arnab Chanda \& McClain, 2019)(Arnab Chanda \& Upchurch, 2018)(Arnab Chanda \& Unnikrishnan, 2015b)(Arnab Chanda \& Unnikrishnan, 2015d)(Arnab Chanda \& Unnikrishnan, 2015c)(Arnab Chanda \& Unnikrishnan, 2015a). This information would be indispensable for fabrication of biofidelic test dummies for automotive crash testing, testing of lethal and non-lethal ballistics, personal protection equipment (PPE) testing in military and contact sports. Also, prosthetic and cosmetic implant industry experts can test the surrogates for improvement in the material comfort and aesthetics of products developed in the future for end consumers. Additionally, these synthetic biomaterials can potentially replace live tissues used for surgical training in clinical settings, requiring almost no biosafety approvals. The major audience for this subject would be research experts in the automotive and sports equipment industry, prosthetics and orthotics, and in the military. Doctors and medical practitioners can also use this information for improved surgical training. Also, a growing community of academic researchers working on soft tissue biomechanics would be able to take advantage of this knowledge for replacing live tissues used in testing, and thereby reducing the cost and time associated with lab.

\section{Materials and Methods}

Discuss about experimental setup, material characterization steps, and hyperelastic models for curve-fitting.

\section{Results and Discussion}

Key experimental results from one paper for each tissue. We can discuss further on this.

\section{Conclusion and Future Directions}

\section{Acknowledgement}

AC acknowledges...

\section{Declaration}

We declare no conflict of interest

\section{References}

Beschorner, K. E., Siegel, J. L., Hemler, S. L., Sundaram, V. H., Chanda, A., Iraqi, A., Haight, J. M., \& Redfern, M. S. (2020). An observational ergonomic tool for assessing the worn condition of slip-resistant shoes. Applied Ergonomics. https://doi.org/10.1016/j.apergo.2020.103140

Chanda, A., \& Ghoneim, H. (2015). Pumping potential of a two-layer left-ventricle-like flexible-matrix-composite structure. Composite Structures. https://doi.org/10.1016/j.compstruct.2014.11.069

Chanda, Arnab;, Unnikrishnan, V., Lackey, K., \& Robbins, J. (2019). Biofidelic conductive 
skin simulant (Patent No. US2019/0057624 A1).

Chanda, Arnab. (n.d.). Customized Human Skin Simulants. 1-11.

Chanda, Arnab. (2017). BIOFIDELIC SOFT COMPOSITES - EXPERIMENTAL AND COMPUTATIONAL MODELING.

Chanda, Arnab. (2018a). Biomechanical Modeling of Human Skin Tissue Surrogates. Biomimetics. https://doi.org/10.3390/biomimetics3030018

Chanda, Arnab. (2018b). Review of Recent Advances in Vaginal Mesh Tissue Interaction. Research \& Development in Material Science, 5(1), 3-5. https://doi.org/10.31031/rdms.2018.05.000601

Chanda, Arnab, \& Callaway, C. (2018). Tissue anisotropy modeling using soft composite materials. Applied Bionics and Biomechanics. https://doi.org/10.1155/2018/4838157

Chanda, Arnab, Callaway, C., Clifton, C., \& Unnikrishnan, V. (2018). Biofidelic human brain tissue surrogates. Mechanics of Advanced Materials and Structures. https://doi.org/10.1080/15376494.2016.1143749

Chanda, Arnab, Chatterjee, S., \& Gupta, V. (2020). Soft composite based hyperelastic model for anisotropic tissue characterization. Journal of Composite Materials.

Chanda, Arnab, \& Curry, K. (2018). Patient-specific biofidelic human coronary artery surrogates. Journal of Mechanics in Medicine and Biology. https://doi.org/10.1142/S0219519418500495

Chanda, Arnab, Flynn, Z., \& Unnikrishnan, V. (2018). BIOMECHANICAL CHARACTERIZATION of NORMAL and PROLAPSED VAGINAL TISSUE SURROGATES. Journal of Mechanics in Medicine and Biology. https://doi.org/10.1142/S0219519417501007

Chanda, Arnab, \& Graeter, R. (2018). Human Skin-Like Composite Materials for Blast Induced Injury Mitigation. Journal of Composites Science. https://doi.org/10.3390/jcs2030044

Chanda, Arnab, Graeter, R., \& Unnikrishnan, V. (2015). Effect of blasts on subject-specific computational models of skin and bone sections at various locations on the human body. AIMS Materials Science. https://doi.org/10.3934/matersci.2015.4.425

Chanda, Arnab, Jones, T. G., \& Beschorner, K. E. (2018). Generalizability of Footwear Traction Performance across Flooring and Contaminant Conditions. IISE Transactions on Occupational Ergonomics and Human Factors. https://doi.org/10.1080/24725838.2018.1517702

Chanda, Arnab, \& McClain, S. (2019). Mechanical Modeling of Healthy and Diseased Calcaneal Fat Pad Surrogates. Biomimetics. https://doi.org/10.3390/biomimetics4010001

Chanda, Arnab, Meyer, I., Richter, H. E., Lockhart, M. E., Moraes, F. R. D., \& Unnikrishnan, V. (2017). Vaginal changes due to varying degrees of rectocele prolapse: A computational study. Journal of Biomechanical Engineering. https://doi.org/10.1115/1.4037222 
Chanda, Arnab, Reuter, A., \& Beschorner, K. E. (2019). Vinyl Composite Tile Surrogate for Mechanical Slip Testing. IISE Transactions on Occupational Ergonomics and Human Factors. https://doi.org/10.1080/24725838.2019.1637381

Chanda, Arnab, Ruchti, T., \& Unnikrishnan, V. (2018). Computational Modeling of Wound Suture: A Review. In IEEE Reviews in Biomedical Engineering. https://doi.org/10.1109/RBME.2018.2804219

Chanda, Arnab, Ruchti, T., \& Upchurch, W. (2018). Biomechanical Modeling of Prosthetic Mesh and Human Tissue Surrogate Interaction. Biomimetics. https://doi.org/10.3390/biomimetics3030027

Chanda, Arnab, \& Unnikrishnan, V. (2015a). Computational Modeling of the Mechanics of Progression of Anterior Vaginal Prolapse (AVP). 13th U.S. National Congress for Computational Mechanics (USNCCM 13).

Chanda, Arnab, \& Unnikrishnan, V. (2015b). Human Skin-Like Biofidelic Sutures. NanoBio Summit.

Chanda, Arnab, \& Unnikrishnan, V. (2015c). Interrupted Suture Force Estimation for Skin Wound Closure: A Computational Approach. Society of Engineering Science 52nd Annual Technical Meeting.

Chanda, Arnab, \& Unnikrishnan, V. (2015d). Numerical Modeling of Skin Wound Closure with Interrupted Sutures. NanoBio Summit.

Chanda, Arnab, \& Unnikrishnan, V. (2015e). Subject-Specific Computational Study of Blast Induced Traumatic Injuries. Society of Engineering Science 52nd Annual Technical Meeting.

Chanda, Arnab, \& Unnikrishnan, V. (2016). Human Tissue Simulants for Study of Traumatic Brain Injury (TBI). American Society for Composites Thirty-First Technical Conference.

Chanda, Arnab, \& Unnikrishnan, V. (2017). A REALISTIC 3D COMPUTATIONAL MODEL of the CLOSURE of SKIN WOUND with INTERRUPTED SUTURES. Journal of Mechanics in Medicine and Biology. https://doi.org/10.1142/S0219519417500257

Chanda, Arnab, \& Unnikrishnan, V. (2018a). Anisotropic soft composite based hyperelastic model. 33rd Technical Conference of the American Society for Composites 2018. https://doi.org/10.12783/asc33/26119

Chanda, Arnab, \& Unnikrishnan, V. (2018b). Effect of bladder and rectal loads on the vaginal canal and levator ani in varying pelvic floor conditions. Mechanics of Advanced Materials and Structures. https://doi.org/10.1080/15376494.2017.1331629

Chanda, Arnab, \& Unnikrishnan, V. (2018c). Novel insole design for diabetic foot ulcer management. Proceedings of the Institution of Mechanical Engineers, Part H: Journal of Engineering in Medicine. https://doi.org/10.1177/0954411918808330

Chanda, Arnab, \& Unnikrishnan, V. (2019). CUSTOMIZED INSOLES FOR DIABETIC AND PRESSURE ULCERS (Patent No. US 10,172,413 B2).

Chanda, Arnab, Unnikrishnan, V., \& Flynn, Z. (2015). Biofidelic Vaginal Tissue Surrogate 
(Patent No. 62/263,942).

Chanda, Arnab, Unnikrishnan, V., \& Flynn, Z. (2018). Biofidelic Skin Simulant (Patent No. US10049601B2).

Chanda, Arnab, Unnikrishnan, V., Flynn, Z., \& Lackey, K. (2017). Experimental study on tissue phantoms to understand the effect of injury and suturing on human skin mechanical properties. Proceedings of the Institution of Mechanical Engineers, Part H: Journal of Engineering in Medicine. https://doi.org/10.1177/0954411916679438

Chanda, Arnab, Unnikrishnan, V., \& Lackey, K. (2017). Biofidelic conductive synthetic skin composites. 32nd Technical Conference of the American Society for Composites 2017. https://doi.org/10.12783/asc2017/15197

Chanda, Arnab, Unnikrishnan, V., Lackey, K., \& Robbins, J. (2020). Biofidelic conductive soft tissue surrogates. International Journal of Polymeric Materials and Polymeric Biomaterials. https://doi.org/10.1080/00914037.2018.1552856

Chanda, Arnab, Unnikrishnan, V., Richter, H. E., \& Lockhart, M. E. (2016a). A biofidelic computational model of the female pelvic system to understand effect of bladder fill and progressive vaginal tissue stiffening due to prolapse on anterior vaginal wall. International Journal for Numerical Methods in Biomedical Engineering . https://doi.org/10.1002/cnm.2767

Chanda, Arnab, Unnikrishnan, V., Richter, H. E., \& Lockhart, M. E. (2016b). Computational Modeling of Anterior and Posterior Pelvic Organ Prolapse (POP). February 2017. https://doi.org/10.1115/imece2016-67949

Chanda, Arnab, Unnikrishnan, V., Roy, S., \& Richter, H. E. (2015). Computational Modeling of the Female Pelvic Support Structures and Organs to Understand the Mechanism of Pelvic Organ Prolapse: A Review. In Applied Mechanics Reviews. https://doi.org/10.1115/1.4030967

Chanda, Arnab, \& Upchurch, W. (2018). Biomechanical Modeling of Wounded Skin. Journal of Composites Science. https://doi.org/10.3390/jcs2040069

Ghoneim, H., \& Chanda, A. (2013). Pumping potential of left-ventrical-like flexible matrix composite structure. ICCM International Conferences on Composite Materials, 2013July(July 2013), 7457-7464.

Gupta, S., Raghuwanshi, G. S., \& Chanda, A. (2020). Effect of weather on COVID-19 spread in the US: A prediction model for India in 2020. Science of the Total Environment. https://doi.org/10.1016/j.scitotenv.2020.138860

Kushner, R. F. (1992). Bioelectrical impedance analysis: A review of principles and applications. In Journal of the American College of Nutrition.

Sundaram, V. H., Hemler, S. L., Chanda, A., Haight, J. M., Redfern, M. S., \& Beschorner, K. E. (2020). Worn region size of shoe outsole impacts human slips: Testing a mechanistic model. Journal of Biomechanics. https://doi.org/10.1016/j.jbiomech.2020.109797 